\title{
Editorial on the Topical Issue “Neutron Biological Physics"
}

\author{
Giovanna Fragneto ${ }^{1}$ and Frank Gabel ${ }^{1,2}$ \\ 1 Institut Laue-Langevin, Grenoble, France \\ ${ }^{2}$ Institut de Biologie Structurale, CEA-CNRS-UJF, Grenoble, France
}

Received 7 June 2013

Published online: 30 July 2013 - (C) EDP Sciences / Società Italiana di Fisica / Springer-Verlag 2013

This special issue brings together a number of original research papers focused on recent developments in biological membrane and membrane model studies by a variety of state-of-the-art biophysical methods. Neutron scattering is especially well represented. Our knowledge of biological macromolecules and their interactions is based on the application of physical methods, ranging from classical thermodynamics to recently developed techniques for the detection and manipulation of single molecules. The role of neutron diffraction and spectroscopy techniques in improving this knowledge has been outstanding and the potential for future developments is remarkable. Many challenges remain for neutron techniques to answer the questions raised by molecular and cellullar biology. The following pages give a flavour of the progress in the field.

A report [1] describes time-resolved small angle scattering of light-induced changes in chloroplast thylakoid membranes. In the context of surface functionalization, the structure in different ionic conditions of sodium hyaluronan grafted at a solid-liquid interface was observed by neutron reflectivity [2]. A review of membrane protein studies [3] addresses the difficulties encountered by providing information on the properties of surfactants used to solubilise membrane proteins for contrast variation studies by neutron small angle scattering, as well as on membrane protein deuterium labelling strategies. The thought-provoking Zaccai neutron resilience and site-specific hydration dynamics in a globular protein are discussed in [4]. Membrane raft model structures with tuneable compositions were characterised by neutron diffraction and reflectivity [5], while raft dynamics in large uni-lamellar vesicles in interaction with beta amyloid peptide was observed by the neutron spin echo method combined with small angle scattering [6]. The bending stiffness of biological membranes as determined by neutron spin echo is also presented [7]. Two contributions [8,9] report on undulation dynamics and shape fluctuations, respectively, in lipid vesicles as a function of lipid composition and other parameters observed by neutron spin echo in combination with other methods like small angle scattering and light scattering. Elastic incoherent neutron scattering was applied to compare dynamics in natural membranes extracted from hyperthermophile and mesophile bacteria in the context of their different biological activities [10]. A molecular dynamics simulation study discusses macromolecular resilience as was defined from neutron spectroscopy experiments. The underlying mechanisms of bioprotection by sugars were explored by using data from Infrared Spectroscopy, Molecular Dynamics simulations, Small Angle X-ray Scattering and Calorimetry [11]. Time resolved small angle X-ray scattering in combination with other biophysical methods can contribute immensely to the study of protein dynamics and a paper presents a new set-up, including a microfluidic continuous-flow mixer, which provided results on unfolding dynamics. And the project of a neutron dynamics data bank is presented in a Perspectives article [12]. The aim of the bank will be to make neutron dynamics data on biological systems widely available to the scientific community.

A variety of questions remain open in molecular and cellular biology. For example, how proteins fold in cellulo. The role of lipids is of paramount importance and important questions include why in Archaea lipids are chemically different from all the commonly encountered species in Bacteria and Eukarya; why is segregation of lipids natural as opposed to complete mixing; to cite only a few. There is a growing need to look at physics on the scales of the cell and interactions of biological macromolecules and their complexes. In the biology of the future, physics should play a major role. Neutrons need to be put in the right context to fully exploit their potential to help unravelling the secrets of life. 
This issue is dedicated to the exceptional career of Joe Zaccaï in neutron biological physics with thanks for his great contribution to neutron scattering in biology. Coming originally from a background in physics, Joe Zaccaï has been working for many decades in the field of molecular and cellular biology. His important scientific contributions as well as his passionate teachings at conferences and in university courses provide an encouraging example for present and future generations of scientists that working at the interface between physics and biology can be very fruitful and rewarding if both disciplines are embraced with passion and open-mindedness.

\section{References}

1. Gergely Nagy, László Kovács, Renáta Ünnep, Ottó Zsiros, László Almásy, László Rosta, Peter Timmins, Judith Peters, Dorthe Posselt, Győző Garab, Eur. Phys. J. E 36, 69 (2013).

2. Ida Berts, Giovanna Fragneto, Jöns Hilborn, Adrian R. Rennie, Eur. Phys. J. E 36, 70 (2013).

3. Cécile Breyton, Frank Gabel, Mathilde Lethier, Ali Flayhan, Grégory Durand, Jean-Michel Jault, Céline Juillan-Binard, Lionel Imbert, Martine Moulin, Stéphanie Ravaud, Michael Härtlein, Christine Ebel, Eur. Phys. J. E 36, 71 (2013).

4. Yinglong Miao, Liang Hong, Zheng Yi, Jeremy C. Smith, Eur. Phys. J. E 36, 72 (2013).

5. V. Rondelli, E. Del Favero, S. Motta, L. Cantù, G. Fragneto, P. Brocca, Eur. Phys. J. E 36, 73 (2013).

6. Mitushiro Hirai, Ryota Kimura, Kazuki Takeuchi, Masaaki Sugiyama, Kouji Kasahara, Noboru Ohta, Bela Farago, Andreas Stadler, Giuseppe Zaccai, Eur. Phys. J. E 36, 74 (2013).

7. Michael Mell, Lara H. Moleiro, Yvonne Hertle, Peter Fouquet, Ralf Schweins, Iván López-Montero, Thomas Hellweg, Francisco Monroy, Eur. Phys. J. E 36, 75 (2013).

8. David J.E. Callaway, Bela Farago, Zimei Bu, Eur. Phys. J. E 36, 76 (2013).

9. B. Brüning, R. Stehle, P. Falus, B. Farago, Eur. Phys. J. E 36, 77 (2013).

10. J. Peters, M.T. Giudici-Orticoni, G. Zaccai, M. Guiral, Eur. Phys. J. E 36, 78 (2013).

11. S. Giuffrida, G. Cottone, G. Bellavia, L. Cordone, Eur. Phys. J. E 36, 79 (2013).

12. Leonid Rusevich, Victoria García Sakai, Bruno Franzetti, Mark Johnson, Francesca Natali, Eric Pellegrini, Judith Peters, Jörg Pieper, Martin Weik, Giuseppe Zaccai, Eur. Phys. J. E 36, 80 (2013). 